\title{
Agradecimento aos Revisores da RBMFC em 2020
}

\section{Acknowledgment of all those who reviewed for RBMFC in 2020}

\section{Reconocimiento a los revisores de la RBMFC en 2020}

\section{Claunara Schilling Mendonça ${ }^{1}$, Leonardo Ferreira Fontenelle ${ }^{1}$, Patricia Sampaio Chueiri ${ }^{1}$, Thiago} Dias Sarti',

${ }^{1}$ Revista Brasileira de Medicina de Família e Comunidade

A Revista Brasileira de Medicina de Família e Comunidade (RBMFC) agradece aos Avaliadores listados abaixo que atuaram como revisores ad hoc durante o ano de 2020, dedicando horas voluntariamente para a emissão de pareceres técnicos sobre manuscritos submetidos a esta revista.

1. Adelson Jantsch / Fundação para o Desenvolvimento Científico e Tecnológico em Saúde - Rio de Janeiro

2. Airton Tetelbom Stein / Universidade Federal de Ciências da Saúde de Porto Alegre (UFCSPA).

3. Alberto Novaes Ramos Júnior / Universidade Federal do Ceará (UFC)

4. Alcione Tavora Kullok / Grupo de Direitos Humanos e Saúde Helena Besserman (DIHS- ENSP)

5. Alessandro da Silva Scholze / Universidade do Vale do Itajaí (UNIVALI)

6. Alexandre José de Melo Neto / Universidade Federal da Paraíba (UFPB)

7. Aline Guerra Aquilante / Universidade Federal de São Carlos (UFSCar)

8. Alisson Oliveira dos Santos / Universidade Federal de Ouro Preto (UFOP)

9. Almária Mariz Batista / Universidade Federal do Rio Grande do Norte (UFRN)

10. Amaury Lelis Dal-Fabbro / Universidade de São Paulo (USP)

11. Ana Rosa Murad Szpilman / Universidade Vila Velha (UVV)

12. Angela Jornada Bem / Vrije Universiteit Amsterdam

13. Anne Jaquelyne Roque Barrêto / Universidade Federal da Paraíba (UFPB)

14. Armando Henrique Norman / Universidade Federal de Santa Catarina (UFSC)

15. Brena de Aguiar Leite / Universidade Federal de Pernambuco (UFPE)

16. Bruna Khayat / Universidade Federal da Paraíba (UFPB)

17. Bruna Moretti Luchesi / Universidade Federal de Mato Grosso do Sul (UFMS)

18. Camila Vescovi Lima / Unimed Vitória

19. Carla Maria Ramos Germano / Universidade Federal de São Carlos (UFSCar)

20. Carlos André Aita Schmitz / Universidade Federal do Rio Grande do Sul (UFRGS)

21. Carlos Frederico Confort Campos / Universidade de São Paulo (USP)

22. Carlos Santos / Universidade Federal de Pernambuco (UFPE) 
23. Carolina Lopes de Lima Reigada / Universidade Estadual do Rio de Janeiro (UERJ)

24. Carolina Maria do Carmo Alonso / Universidade Federal do Rio de Janeiro (UFRJ)

25. Célia Aparecida Paulino / Eikon University (Brasil)

26. Charles Dalcanale Tesser / Universidade Federal de Santa Catarina (UFSC)

27. Claunara Schilling Mendonca / Universidade Federal do Rio Grande do Sul (UFRGS)

28. Daniel Moreira Pinto / Universidade Federal dos Vales do Jequitinhonha e Mucuri (UFVJM)

29. Daniel Knupp Augusto / UNIMED, Belo Horizonte, MG

30. Daniela Dallegrave / Universidade Federal de Goiás (UFG)

31. Daniela Koeller Rodrigues Vieira / Fundação de Saúde de Angra dos Reis

32. David Ramos da Silva Avigo / Universidade Federal da Bahia (UFBA)

33. Debora Carvalho Ferreira / Universidade Federal de Viçosa (UFV)

34. Débora Gusmão Melo / Universidade Federal de São Carlos (UFSCar)

35. Deise Fernanda Peixoto Oliveira / Universidade Federal de Sergipe (UFS)

36. Deoclecio Avigo / Faculdade de Medicina da Universidade de São Paulo. Campus SP

37. Diana de Oliveira Frauches / Escola Superior de Ciências da Santa Casa de Misericórdia de Vitória

38. Diego José Brandão / Universidade Vila Velha (UVV)

39. Donovan Casas Patiño / Centro Universitario Amecameca de la Universidad Autnoma del Estado de Mexico y Medico Familiar del Instituto Mexicano del Seguro Social

40. Eduardo Alexander Júlio César Fonseca Lucas / Universidade Federal do Rio de Janeiro (UFRJ)

41. Eduardo Bertol / Secretaria Municipal de Saúde de Curitiba

42. Eno Dias de Castro Filho / Grupo Hospitalar Conceição (GHC)

43. Enrique Falceto Barros / Universidade de Caxias do Sul

44. Fabio Fortunato Brasil de Carvalho / Instituto Nacional de Câncer José Alencar Gomes da Silva (INCA)

45. Fabíola Fernandes Bersot Magalhães / Universidade Federal do Espírito (UFES)

46. Fatima Aparecida Ferreira Teixeira de Carvalho / Universidade de Ribeirão Preto (UNAERP)

47. Felipe Monte Cardoso / Universidade Estadual de Campinas (UNICAMP)

48. Felipe Proenço / Universidade Federal da Paraíba (UFPB)

49. Ferla Maria Simas Bastos Cirino / Prefeitura Municipal de Diadema

50. Fernanda Pereira de Paula Freitas / Faculdade de Medicina da UFRJ

51. Flavio Borges Nedel / Rede de Pesquisa em Atenção Primaria à Saúde (REDE APS)

52. Francisco Eduardo da Fonseca Delgado / Universidade do Porto, Portugal / Pesquisador Convidado - Universidade do Estado do Rio de Janeiro, Brasil / Professor Pesquisador Bolsista Instituto Federal de Educação, Ciência e Tecnologia do Sul de Minas Gerais- Campus Muzambinho - CEAD/e-tec/FNDE/MEC

53. Francisco Jorge Arsego Quadros de Oliveira / Universidade Federal do Rio Grande do Sul (UFRGS)

54. Gabriela Mardero / Universidade Federal do Ceará (UFC)

55. Giannina do Espírito-Santo / Centro Universitário Augusto Motta (UNISUAM)

56. Giovana Andrade Frederico / Universidade Federal de São Paulo (UNIFESP)

57. Glenda Blaser Petarli / Universidade Federal do Espirito Santo (UFES)

58. Graciano Almeida Sudré / Universidade Federal de Rondonópolis (UFR)

59. Gustavo Diniz Ferreira Gusso / Universidade de São Paulo (USP)

60. Gustavo Gonçalves dos Santos / Centro Universitário das Faculdades Metropolitanas Unidas FMU

61. Heloisa Taboada / Secretaria Municipal de Saúde do Rio de Janeiro

62. Henriqueta Sacramento / Prefeitura Municipal de Vitória

63. Hernâni Pombas Caniço / Faculdade de Medicina da Universidade de Coimbra

Como citar: Sarti TD, Fontenelle LF. Agradecimento aos Revisores da Revista Brasileira de

Medicina de Família e Comunidade(RBMFC) que avaliarammanuscritosem2020. Rev Bras Med

Fam Comunidade. 2020;15(42):2861. http://doi.org/10.5712/rbmfc15(42)2861 
64. Hilmar Dias Ricardo / Universidade do Estado do Rio de Janeiro (UERJ)

65. Isabel Brandão Correia / Secretaria de Saúde do Recife

66. Italla Maria Bezerra / Escola Superior de Ciências da Santa Casa de Misericórdia de Vitória

67. Jacqueline Ponzo / Sociedad Uruguaya de Medicina Familiar y Comunitaria

68. Janaina Alves da Silveira Hallais / Universidade Estadual de Campinas (UNICAMP)

69. Jeferson Gervasio Pires / Universidade Federal de Santa Catarina (UFSC)

70. João Mazzoncini de Azevedo Marques / Universidade de São Paulo (USP)

71. Johnnatas Mikael Lopes / Universidade Federal do Vale do São Francisco (UNIVASF)

72. Josenaide Engracia Santos / Universidade de Brasília (UnB)

73. Juliana da Rosa Wendt / Universidade Federal de Santa Maria (UFSM)

74. Julie Silvia Martins / Atenção Primaria à Saúde Santa Marcelina

75. Lais Santos Vitti / Pontifícia Universidade Católica de Campinas (PUC-Campinas)

76. Leandro David Wenceslau / Universidade Federal de Viçosa (UFV)

77. Leonardo Cançado Monteiro Savassi / Universidade Federal de Ouro Preto - UFOP e Universidade Federal de Minas Gerais - UFMG

78. Leonardo Ferreira Fontenelle / Universidade Vila Velha (UVV)

79. Leonardo Moscovici / Universidade de São Paulo (USP)

80. Letícia de Castro Martins Ferreira / Universidade Federal de Juiz de Fora (UFJF)

81. Lourdes Luzón Oliver / Secretaria Municipal de Saúde do Rio de Janeiro (SMS-RJ)

82. Lucas Gaspar Ribeiro / Universidade de São Paulo de Ribeirão Preto (FMRB-USP)

83. Lucas Wollmann / Grupo Hospitalar Conceição - Porto Alegre/RS

84. Lucas Alexandre Pedebôs / Prefeitura Municipal de Florianópolis (PMF)

85. Luccas Salvador Salomão / Santa Casa de Misericórdia de Porto Alegre

86. Lucia Alves S. Lara / Universidade de São Paulo de Ribeirão Preto (FMRB-USP)

87. Luciano Nunes Duro / Universidade de Santa Cruz do Sul, UNISC, RS e Prefeitura Municipal de Santa Cruz do Sul, RS.

88. Luís Antônio Benvegnú / Universidade Regional do Noroeste do Estado do Rio Grande do Sul (UNIJUI)

89. Luiz Felipe da Silva da Silva / Universidade Federal do Rio de Janeiro (UFRJ)

90. Marcello Dala Bernardina Dalla / Universidade Vila Velha (UVV)

91. Marcelo Pellizzaro Dias Afonso / Universidade Federal de Minas Gerais (UFMG)

92. Marcelo Santana Vetis / Universidade Vila Velha (UVV)

93. Márcia Dornelles Machado Mariot / Universidade Federal do Rio Grande do Sul (UFRGS)

94. Marcos Krahe Edelweiss / Clinica Médica Santa Úrsula, Florianópolis

95. Margarita Silva Diercks / Hospital Nossa Senhora da Conceição-GHC (CEPAPS-SSC-GHC)

96. Maria Beatriz Junqueira Camargo / Universidade Federal de Pelotas (UFPel)

97. Maria Inez Padula Anderson / Universidade do Estado do Rio de Janeiro (UERJ)

98. Marilia Jesus Batista / Faculdade de Medicina de Jundiaí

99. Mário Roberto Tavares Cardoso de Albuquerque Albuquerque / Centro Universitário do Pará (CESUPA)

100. Mario Maia Bracco / Instituto Israelita de Ensino e Pesquisa Albert Einstein (IIEPAE). São Paulo, SP

101. Maristela Carbol / Universidade Federal de São Carlos (UFSCar)

102. Maurício Moraes / Universidade Federal de Rio Grande (FURG)

103. Melanie Noël Maia / Secretaria Municipal de Saúde do Rio de Janeiro (SMS-RJ)

104. Miguel Pizzanelli Báez / Facultad de Medicina de la Universidad de la República (UDELAR), Uruguay

105. Milena Andrade Baiano / Universidade Federal de Sergipe (UFS)

106. Murilo Moura Sarno / Universidade Municipal de são Caetano do Sul (USCS) 
107. Nathan Mendes Souza / Universidade Federal de Minas Gerais (UFMG)

108. Nelson Robson Mendes de Souza / Universidade Estadual do Rio de Janeiro (UERJ)

109. Nicole Geovana Dias Carneiro / Universidad de Alicante

110. Nilson Massakazu Ando / Secretaria Municipal de Saúde de Manaus (SEMSA)

111. Nivaldo Carneiro Júnior / Faculdade de Ciências Médicas da Santa Casa de são Paulo

112. Olivan Queiroz / Universidade Federal de São Paulo (Unifesp)

113. Omaira Flores Martínez / Sociedad Venezolana de Medicina Familiar (SOVEMEFA).

114. Patricia Barreto Cavalcanti / Universidade Federal da Paraíba (UFPB). João Pessoa, PB

115. Patricia Sampaio Chueiri / Faculdade Israelita de Ciências da Saúde Albert Einstein

116. Patrick Harris de Mesquita / Universidade de São Paulo (USP)

117. Paulo Poli Neto / Universidade Federal do Paraná (UFPR)

118. Pedro Igor Daldegan De Oliveira / Universidade Estadual do Rio de Janeiro (UERJ)

119. Pedro Walisson Gomes Feitosa / Universidade Federal do Cariri (UFCA)

120. Rafael Nicolau Carvalho / Universidade Federal da Paraíba (UFPB)

121. Randson Rosa / Universidade Estadual do Sudoeste da Bahia (UESB)

122. Raquel Ferreira / Instituto René Rachou, Fundação Oswaldo Cruz

123. Regis Rodrigues Vieira / Hospital Albert Einstein

124. Renata Cereda Cordeiro /Universidade Federal de São Paulo (UNIFESP)

125. Ricardo Alexandre de Souza / Universidade Federal de Minas Gerais (UFMG)

126. Ricardo Souza Heinzelmann / Universidade Federal de Santa Maria (UFSM)

127. Rinaldo Eduardo Machado de Oliveira / Faculdade de Medicina de Ribeirão Preto da Universidade de São Paulo (FMRP-USP)

128. Roberta Alvarenga Reis / Universidade Federal do Rio Grande do Sul (UFRGS)

129. Roberta Fonseca Petrocchi / Associação Capixaba de Medicina da Família e Comunidade

130. Rodolfo Rêgo Deusdará Rodrigues / Universidade de Brasília (UNB)

131. Rogerio Luz Coelho / Faculdade Evangélica de Medicina do Paraná (FEPAR)

132. Rosália Garcia Neves / Universidade Federal de Pelotas

133. Rosana Pimentel Correia Moysés / Universidade Federal do Amazonas (UFAM)

134. Rosangela Ziggiotti Oliveira / Universidade Estadual de Maringá (UEM)

135. Rosimere de Jesus Teixeira / Universidade do Estado do Rio de Janeiro (UERJ)

136. Rubens Carvalho Rubão / Prefeitura Municipal de Aracaju

137. Sandra Fortes / Universidade Estadual do Rio de Janeiro (UERJ)

138. Sérgio Henrique de Oliveira Botti / Universidade Federal do Estado do Rio de Janeiro (UNIRIO)

139. Tânia de Araujo Barboza / Universidade de Fortaleza (UNIFOR)

140. Thiago Dias Sarti / Universidade Federal do Espírito Santo (UFES)

141. Thiago Santos Souza / Universidade Federal da Bahia (UFBA)

142. Valéria Ferreira Romano / Universidade Federal do Rio de Janeiro (UFRJ)

143. Welington Serra Lazarini / Universidade Federal do Espírito Santo, Vitória (UFES)

144. Willian Luna / Universidade Federal de São Carlos (UFSCar)

145. Yuji Magalhães Ikuta / Universidade do Estado do Pará (UEPA) 